\title{
Le capital ferroviaire britannique, entre patrimoine et pragmatisme
}

The capital of British rail: between heritage and pragmatism

Das britische Eisenbahnerbe zwischen Erbe und Pragmatismus

\section{Anne Hecker}

\section{(2) OpenEdition}

\section{Journals}

Édition électronique

URL : http://journals.openedition.org/rge/1241

DOI : $10.4000 /$ rge. 1241

ISSN : $2108-6478$

Éditeur

Association des géographes de l'Est

Édition imprimée

Date de publication : 1 janvier 2008

ISSN : 0035-3213

\section{Référence électronique}

Anne Hecker, « Le capital ferroviaire britannique, entre patrimoine et pragmatisme », Revue

Géographique de l'Est [En ligne], vol. 48 / 1-2 | 2008, mis en ligne le 08 octobre 2011, consulté le 08 septembre 2020. URL : http://journals.openedition.org/rge/1241; DOI : https://doi.org/10.4000/rge. 1241

Ce document a été généré automatiquement le 8 septembre 2020

Tous droits réservés 


\title{
Le capital ferroviaire britannique, entre patrimoine et pragmatisme
}

\author{
The capital of British rail: between heritage and pragmatism \\ Das britische Eisenbahnerbe zwischen Erbe und Pragmatismus
}

\section{Anne Hecker}

1 La Grande-Bretagne est l'Etat européen ferroviaire par excellence. De l'ouverture de la toute première liaison ferrée en 1825 , aux progrès techniques et aux records de vitesse, elle a longtemps affiché sa suprématie dans ce domaine. Néanmoins, d'autres facteurs certifient le caractère novateur de la Grande-Bretagne dans le monde ferroviaire. La réalisation de son réseau s'est ainsi avérée précoce : dès 1850 , alors qu'à peine $8 \%$ des futures lignes françaises sont construites, $32 \%$ des liaisons voyageurs britanniques sont déjà ouvertes, et forment un réseau connexe, irriguant l'essentiel du territoire. Vingt ans plus tard, $70 \%$ des voies ferrées britanniques sont achevées, contre $40 \%$ sur le territoire français. Au total, son exceptionnelle densité ferroviaire atteint 15,6 km de voie ferrée pour $100 \mathrm{~km}^{2}\left(8,1 \mathrm{~km} / 100 \mathrm{~km}^{2}\right.$ en France). En Europe, seules la Belgique et l'Allemagne seront à même de rivaliser. La contraction ferroviaire britannique apparaît également précocement. Une première liaison ferme au trafic des voyageurs dès 1846, après seulement 12 années de service, un demi-siècle avant la première disparition d'une liaison française. La contraction va s'avérer non seulement rapide - en 1970, 97 \% des lignes voyageurs aujourd'hui disparues sont déjà fermées - mais également quantitativement importante : au total, $53 \%$ du réseau total sera sacrifié, contre $37 \%$ en France ${ }^{1}$.

2 Le mode de constitution, puis de contraction du réseau britannique, expliquent l'importance du capital linéaire aujourd'hui abandonné, mais aussi potentiellement valorisable. Toujours novateurs, les Britanniques ont également été les premiers Européens à mettre en œuvre une réelle valorisation de l'une de ces emprises abandonnées. Ils vont participer à imposer sur le continent le futur concept de voie verte - un espace en site propre dédié à la circulation non motorisée, fréquemment implanté sur une infrastructure de transport déclassée. Pourtant, le patrimoine ferroviaire ne connaît en Grande-Bretagne qu'une mise en valeur limitée et sélective. 
Après une décennie d'avance, la Grande-Bretagne cède du terrain: les projets de Sustrans, principale association en charge de la valorisation des emprises délaissées, s'orientent désormais vers davantage de pragmatisme, au détriment du patrimoine. Le modèle britannique précurseur, à l'origine de multiples aménagements continentaux, s'oppose aujourd'hui aux modèles belge et espagnol, qui demeurent très attachés au principe même de valorisation du patrimoine ferroviaire.

3 Au-delà d'une rivalité, apparemment anodine, visant à imposer une pensée unique en termes de voies vertes, c'est en toile de fond l'avenir du patrimoine ferroviaire déclassé qui pourrait se jouer. La réussite du modèle britannique, qui pourrait tendre à l'imposer comme schéma unique d'aménagement de voies vertes, pourrait impliquer la disparition de la principale opportunité de réhabilitation des anciens itinéraires de transport.

\section{I - Un vaste patrimoine ferroviaire en manque de patrimonialisation}

4 La Grande-Bretagne possède un capital ferroviaire considérable : sur les $35700 \mathrm{~km}$ de lignes construites - ponctués de 9000 gares, 1000 tunnels et 60000 ponts et viaducs seuls $17000 \mathrm{~km}$ demeurent aujourd'hui en service ${ }^{2}$, laissant près de $19000 \mathrm{~km}$ d'emprises à l'abandon. Le réseau britannique a à ce titre enregistré la plus forte contraction des réseaux ferrés européens. Le patrimoine qui en découle ne bénéficie toutefois, en Grande-Bretagne comme ailleurs, que d'une patrimonialisation très sélective, qui exclut largement l'ancienne infrastructure ferrée.

\section{A - Un vaste capital ferroviaire}

5 Les conditions financières, administratives et juridiques qui ont prévalu lors de la mise en place du réseau ferré britannique à l'époque victorienne éclairent l'aspect pléthorique qu'il présentait dans sa plus grande extension (voir figure 1). 
Figure 1 - Réseau ferré britannique : un vaste patrimoine, rarement valorisé

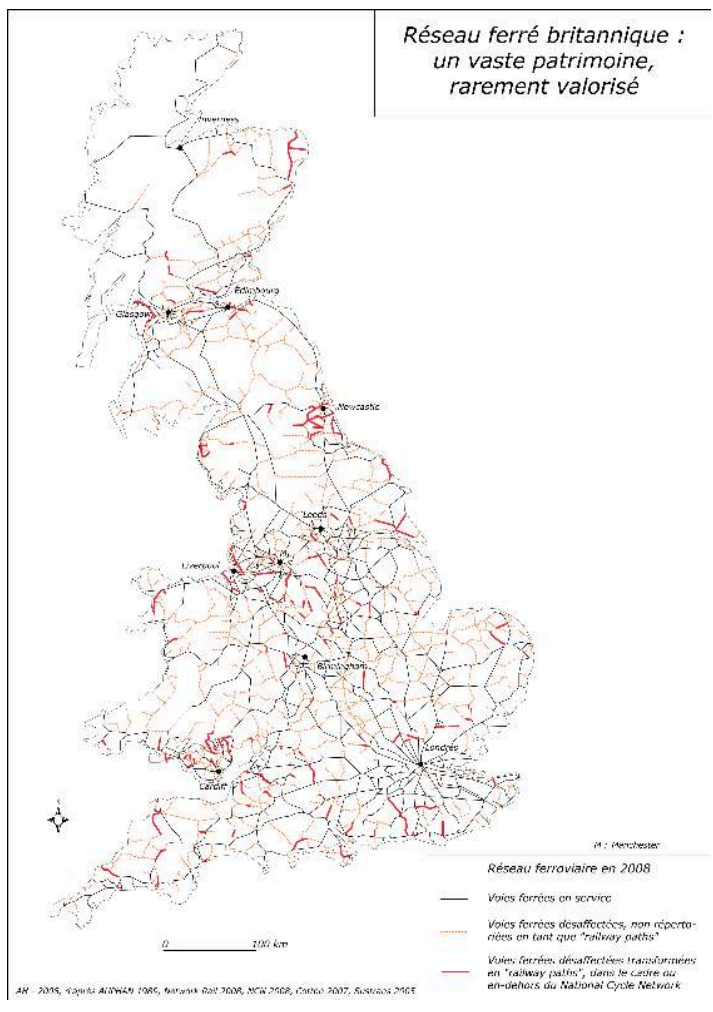

6 Sa réalisation s'effectue en effet dans un climat d'affairisme et de libéralisme économique et industriel, et en l'absence totale d'intervention de l'Etat, jusque dans la conception même du réseau. Certes, la mise à exécution de chaque projet ferroviaire est soumise à l'autorisation législative de l'Etat britannique. Mais là où certains pays s'assurent de la cohérence et de l'efficacité du futur réseau, la Grande-Bretagne se distingue par un laisser-faire absolu. Aucun schéma, dessinant un réseau ferroviaire rationnel, n'est imposé, laissant libre cours à la spéculation des industriels et des financiers, conduisant à la "railway mania». Cette fièvre ferroviaire atteint son paroxysme en 1846, année qui enregistre la création de 272 compagnies ferroviaires, à la tête de $15000 \mathrm{~km}$ de voies ferrées à construire... Nombre de ces projets disparaissent rapidement, mais en 1885 , après une première concentration ferroviaire et avant l'achèvement du réseau, la Grande-Bretagne dénombre 300 compagnies, exploitant $31000 \mathrm{~km}$ de chemin de fer...

7 La spéculation financière donne lieu à la multiplication de liaisons ferrées, principalement dans les secteurs miniers et industriels, particulièrement attractifs. Le triangle Manchester - Leeds - Sheffield, et plus tardivement le Pays de Galles, se couvrent ainsi de voies ferrées (figure 1), le plus souvent redondantes en raison de la concurrence effrénée que se livrent les compagnies, qui n'hésitent pas à ouvrir, au sein d'une même vallée, plusieurs liaisons concurrentes. Ainsi, dans les années 1880, deux compagnies créent chacune une liaison entre Nottingham et Mansfield, établies parallèlement à celle du Midland Railway déjà en service, les trois tenant souvent dans un faisceau d'une centaine de mètres ${ }^{3}$ (figure 2)... Ces multiplications de parcours concurrents concernent également de vastes itinéraires, à l'image de Glasgow Edimbourg, de Liverpool - Manchester ou de Londres - Birmingham (figure 1). 
Figure 2 - Nottingham-Mansfield : l'enchevêtrement des trois voies ferrées au nord de Hucknall La cohabitation sur le terrain de ces trois infrastructures ferrées redondantes, installées dans un faisceau de quelques centaines de mètres à peine, illustre le libéralisme, l'absence de planification du réseau par l'Etat et son laissez-faire qui ont caractérisé la constitution du réseau ferré britannique.

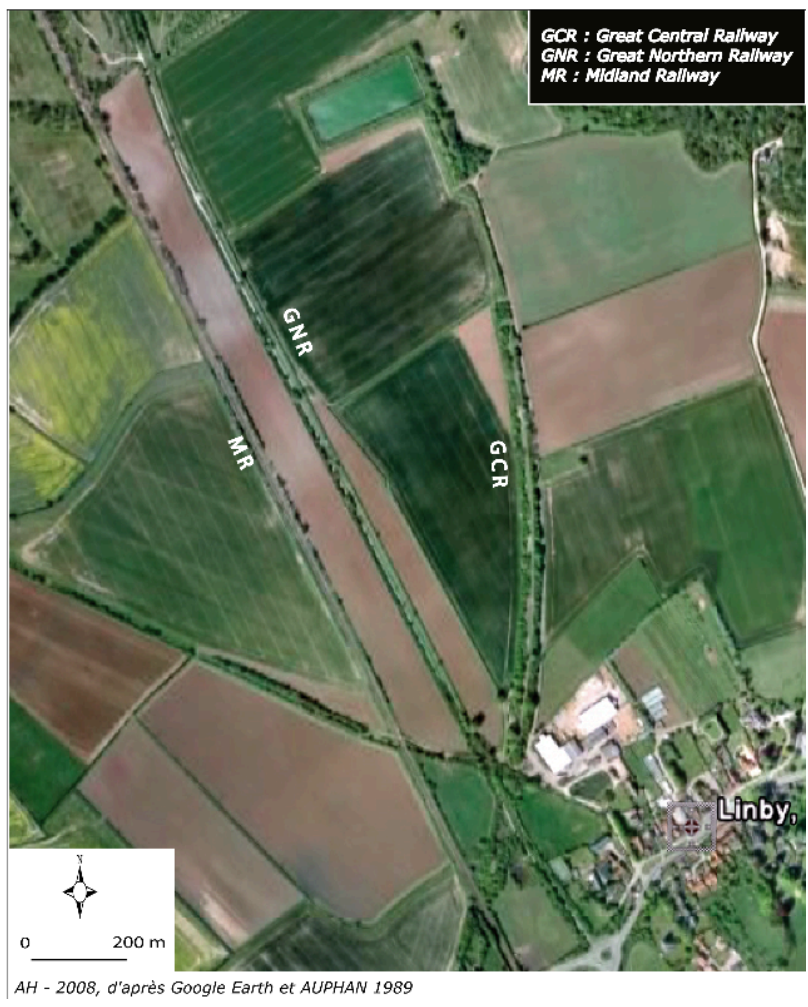

Néanmoins, la trop grande proximité et la redondance des liaisons ainsi créées ne pouvaient assurer la rentabilité de tout le réseau, et rapidement, sa contraction s'impose. Dans un premier temps, les fermetures ne concernent que le seul service des voyageurs, les lignes subsistant pour le trafic de fret. Mais l'implication de l'Etat en faveur d'une réelle rationalisation du réseau ferré va bientôt se faire plus prégnante, et débute par l'unification des compagnies. En 1921, le Grouping Act (appliqué en 1923) regroupe le réseau de 120 compagnies entre les mains des "Big Four », impliquant la disparition de $2000 \mathrm{~km}$ de liaisons redondantes, désormais détenues par une même compagnie. Néanmoins, ce réseau encore trop vaste, désormais concurrencé par les transports routiers, demeure déficitaire. Les compagnies limitent investissements et entretien, amorçant sa dégradation lente et sa désaffection future ${ }^{4}$... et précipitant sa nationalisation en 1948, et avec elle davantage de rationalité. Cinq mille nouveaux kilomètres de voies ferrées sont ainsi rapidement supprimés. Au-delà des liaisons minières et industrielles parallèles, on observe également les premières fermetures de voies non redondantes en milieu rural (figure 1), la rationalisation s'attaquant aux courtes antennes peu fréquentées, qui raccordent de petites localités au réseau principal. On assiste également aux premières suppressions de longs itinéraires, tels que les $150 \mathrm{~km}$ de la ligne du Midland and Great Northern Joint sur la liaison Norwich Nottingham. La contraction du réseau demeure toutefois encore modérée ... une accélération brutale va être donnée avec l'application du Beeching Axe.

Face aux difficultés chroniques des British Railways, le rapport du Dr Beeching en 1963 est sans appel : $96 \%$ du trafic voyageurs est assuré par $50 \%$ du réseau ; l'autre moitié, avec seulement $4 \%$ du trafic, s'avère donc quasi-inutile. "The Reshaping of Bristish 
Railway report » propose donc la fermeture de $9700 \mathrm{~km}$ de voies ferrées, essentiellement en milieu rural. Le Beeching Axe ne sera pas totalement appliqué, et d'autres liaisons, exclues de ce programme, disparaissent malgré tout. Néanmoins, en 1975, des $35700 \mathrm{~km}$ et 9000 gares construits, il ne reste que moins de $20000 \mathrm{~km}$ et 2500 gares. Par la suite, la contraction se concentre majoritairement sur le trafic de fret résiduel. En effet, il est désormais communément admis que la fermeture de lignes de voyageurs ne suffit pas à rééquilibrer les finances de la compagnie ferroviaire. Pire, la disparition des mailles rurales qui irriguaient le territoire détourne du chemin de fer une part non négligeable de la population, ce qui restreint de fait la fréquentation du «réseau noyau ». Par ailleurs, la reconnaissance des services rendus par le chemin de fer justifie désormais le maintien, politiquement et économiquement assisté, de la partie la moins rentable du réseau encore ouvert. En revanche, les liaisons de fret non rentables ne bénéficient pas de la même indulgence et continuent de disparaître.

Aujourd'hui, la contraction du réseau semble proche de la fin, et l'on assiste même à des réouvertures d'infrastructures, notamment en milieu urbain, pourtant moins touché par la contraction. La demande y demeure néanmoins plus forte, et le réseau ferré y a prouvé sa plus grande efficacité et sa meilleure rentabilité. Si les courtes liaisons, voire les services de type tramway ou tram-train dominent, quelques réouvertures plus conséquentes ont pu être menées à bien, et d'autres sont en projet, comme la réouverture de la liaison Edimbourg - Galashiels (56 km), espérée pour 2011.

11 Cette forte expansion du réseau ferré britannique, suivie de son importante contraction, ont offert à la Grande-Bretagne un vaste héritage ferroviaire, qu'elle ne valorise pourtant que de manière sélective.

\section{B - Une patrimonialisation sélective}

12 L'attachement des Britanniques à leur patrimoine ferroviaire est réel, et se traduit notamment par les 8 millions de passagers annuels des trains historiques ou par les 900000 visiteurs qui fréquentent chaque année le musée du chemin de fer de York ${ }^{5}$. Néanmoins, la patrimonialisation ferroviaire exclut, en Grande-Bretagne comme ailleurs, le fondement même du chemin de fer, l'infrastructure ferrée.

Dans la plupart des pays européens, la patrimonialisation ferroviaire ne s'est pas imposée comme une évidence. Les vestiges ferroviaires cumulent en effet divers handicaps, notamment celui d'être un patrimoine récent relevant des $\mathrm{XIX}^{\mathrm{e}}$ et $\mathrm{XX}^{\mathrm{e}}$ siècles, mais aussi d'appartenir au domaine industriel. La sensibilisation à leurs valeurs patrimoniales se révèle très tardive. L'Angleterre présente dans ce domaine une longueur d'avance, avec notamment le classement du célèbre Iron Bridge dès 1934. Situé dans la vallée de Coalbrookdale, berceau des premiers rails métalliques, et où Richard Trevithick testa en 1802 sa locomotive à vapeur, l'Iron Bridge demeure néanmoins étranger au monde ferroviaire, à l'instar de $97 \%$ des sites "listés " britanniques. Néanmoins, cette reconnaissance précoce d'un ouvrage du XIX siècle, relevant $\mathrm{du}$ monde des transports, représente une double avancée importante. Ce dernier relève en effet d'un domaine usuel, quotidien, dont le public et les politiques ne mesurent que partiellement, aujourd'hui encore, l'aspect patrimonial.

La reconnaissance précoce du capital technique, et notamment de la traction vapeur, constitue une exception. Elle a donné naissance à des musées, tels que celui de York, ouvert en 1975 dans sa configuration actuelle, ainsi qu'à de nombreux projets de 
chemins de fer historiques, exploitant ce capital grandeur nature. Quant au patrimoine bâti, il bénéficie, depuis que l'architecture des XIX ${ }^{e}$ siècle puis $\mathrm{XX}^{\mathrm{e}}$ siècle a acquis ses lettres de noblesse, d'une certaine reconnaissance. La destruction en 1962 de l'Arc néoclassique de la gare de Euston a eu en Grande-Bretagne un impact similaire à celui de la démolition des pavillons de Baltard à Paris, à l'origine de la préservation de la gare d'Orsay. Un mouvement de protection, concernant essentiellement les bâtiments et les ouvrages d'art du XIX ${ }^{e}$ siècle, voit alors le jour. Le système législatif britannique offre trois dispositifs de préservation. Le classement à proprement parler (loi de 1979) ${ }^{6}$, ne concerne que 56 monuments ferroviaires, tous aujourd'hui désaffectés, appartenant pour l'essentiel à d'anciens chemins de fer minéraliers à traction hippomobile. Bien plus nombreux sont les bâtiments «listés » ${ }^{7}$. Parmi les 500000 monuments britanniques ainsi reconnus en 2008, 1383 appartiennent au domaine ferroviaire, inclus pour l'essentiel dans des lignes encore en activité. La " protection » accordée par ce processus apparaît toutefois moins contraignante que celle des monuments historiques français. Il s'agit davantage d'une reconnaissance de leur intérêt, autorisant un compromis entre aspect utilitaire et préservation. Enfin, les secteurs protégés ${ }^{8}$ incluent un millier de bâtiments ferroviaires.

Au-delà de English Heritage, de Cadw et de Historic Scotland - en charge du patrimoine classé ou listé -, des organismes sont plus précisément préposés au patrimoine hérité du monde ferroviaire. Depuis 1994, le Railway Heritage Committee protège objets (roulants ou non) et archives ferroviaires. En 1997, suite à la privatisation de l'infrastructure, la partie désaffectée du réseau non encore cédée est affectée au British Railways Board (Residuary) Ltd. Ce dernier et Network Rail financent le Railway Heritage Trust, chargé de la conservation et de la restauration de leur patrimoine ferroviaire en priorité le patrimoine exceptionnel, classé ou listé. Des 3800 structures ferroviaires ponctuant les voies désaffectées prises en charge par le BRB (Residuary) Ltd, 72 sont ainsi listées ${ }^{9}$. Le Railway Heritage Trust gère également le transfert des sites fermés à leurs nouveaux propriétaires et, depuis le milieu des années 1990, participe à retrouver une nouvelle vocation aux bâtiments et structures restaurés par ses soins.

Ces structures ferroviaires protégées représentent essentiellement des bâtiments (gares, halles à marchandises, postes de signalisation, marquises ...), quelques objets techniques, ainsi que des tunnels, des ponts et des viaducs ferroviaires, tels que le Forth Bridge (Ecosse) ou le Brunel Bridge de Saltash (Cornouailles). Mais aucune ne relève de l'emprise à proprement parler. Les actions de ces institutions traduisent en GrandeBretagne une réalité très présente sur tout le continent européen : la protection du patrimoine ferroviaire se concentre en premier lieu sur le patrimoine bâti exceptionnel, à parité avec le matériel roulant, puis sur les éléments d'infrastructures non moins exceptionnels (viaducs et tunnels), et enfin sur les archives. Quant à l'infrastructure « basique », elle ne semble guère retenir l'attention.

\section{C - Quelle reconnaissance pour l'infrastructure?}

17 La patrimonialisation de l'infrastructure ferroviaire se heurte en effet à deux obstacles. Une méconnaissance populaire tout d'abord, tant sa présence semble habituelle, ses qualités ordinaires et son utilité déficiente une fois éteinte sa vocation première. Si la préservation et la valorisation d'un bâtiment de belle facture, même aussi modeste que la petite gare tout en verre et en lignes courbes de Wemyss Bay (Ecosse), ou d'un portail de tunnel spectaculaire tel celui de Clayton (Londres - Brighton) ne posent plus 
réellement question aujourd'hui, les efforts pour préserver les emprises désaffectées, simples rubans de terre, demeurent pour beaucoup incompréhensibles. Ce déni d'intérêt n'a pas permis de remettre en cause l'obstacle législatif, qui renforce cette absence de reconnaissance : en Grande-Bretagne, seul un espace bâti peut être reconnu et protégé. Cela autorise la patrimonialisation des gares, des halles à marchandises, des postes de signalisation ... mais pas de l'emprise elle-même, à l'exception des tunnels, des ponts et des viaducs, assimilés à des constructions. Ainsi, l'exception exemplaire de la ligne française du Blanc-Argent, inscrite à l'inventaire supplémentaire des monuments historiques en 1993, serait actuellement impossible en Grande-Bretagne. Seule l'intégration au sein d'un secteur protégé peut placer une section de ligne dans un cadre patrimonial, mais sans aucune garantie de sa réelle préservation, ni de la conservation de son intégralité. Surtout, aucune réelle reconnaissance de la voie ferrée en tant que patrimoine à part entière ne lui est alors accordée. La Grande-Bretagne est donc loin de la politique américaine de railbanking qui veut que, même valorisée ou transformée, l'infrastructure « mise en banque » demeure inaliénable et ne puisse être dégradée (par la suppression d'un ouvrage d'art ou la construction d'un bâtiment dans l'axe de l'emprise par exemple), afin que soit préservés sa continuité et sa linéarité, garants d'une potentielle réouverture.

Ces infrastructures ne sont pourtant pas dénuées d'atouts. Témoins de l'évolution culturelle, industrielle et paysagère des $\mathrm{XIX}^{\mathrm{e}}$ et $\mathrm{XX}^{\mathrm{e}}$ siècles et jalons de la révolution industrielle, elles ont nécessité la mise en pratique de techniques nouvelles (terrassement, excavation, conception d'ouvrages aériens ...). Par leur présence, les voies ferrées ont bouleversé les paysages, notamment industriels mais aussi urbains et périurbains, et constituent autant de jalons de l'histoire économique et sociale des territoires traversés.

19 Au-delà de l'objet de mémoire, une emprise préservée constitue également un capital pour l'avenir ferroviaire de sa région. Les réouvertures actuelles se trouvent simplifiées lorsque le délaissé a été sauvegardé. Ainsi, le projet de nouvelle liaison rapide entre l'East Anglia et le sud, le centre et l'ouest de l'Angleterre est remis en cause par la disparition partielle de l'emprise de la Varsity Line (Oxford - Cambridge) : si la partie occidentale (Oxford - Bletchley), peu endommagée, devrait rouvrir en 2012, moyennant 190 millions de Livres Sterling, les plus optimistes n'évaluent qu'à 2028 l'éventuelle réouverture de la partie orientale, partiellement disparue, que d'aucuns considèrent comme définitivement perdue ...

Pendant des décennies, la compagnie ferroviaire britannique, peu consciente de cet intérêt patrimonial, s'est rapidement dessaisie de ses anciennes emprises, débitées en courts tronçons cédés aux riverains. Une récente prise de conscience patrimoniale a conduit la $B R B(R) L t d$ à en rechercher prioritairement la cession d'un seul tenant, et a trouvé un écho favorable des collectivités locales, désormais soucieuses de protéger ce capital et de le valoriser, notamment sous la forme de sentiers ruraux. A ce titre, la Grande-Bretagne bénéficie d'une spécificité juridique, qui a permis de préserver, au moins partiellement, la linéarité de nombreux délaissés. Le principe du «Right-ofWay " multiplie en effet les sentiers ruraux, en accordant aux piétons, voire aux cyclistes ou aux cavaliers, un droit de passage sur les propriétés privées, garanti par la loi après qu'un accord a été passé entre le propriétaire et les autorités locales. Certains délaissés ont ainsi été transformés en sentiers, sans pour autant bénéficier de l'entretien qui permettrait de les considérer comme réellement valorisés. Ces droits de 
passage maintiennent toutefois la trace de l'emprise, et rendent possible son éventuelle mutation ultérieure en chemin aménagé, notamment sous l'influence de Sustrans.

\section{II - Sustrans, la réussite d'un système précurseur}

21 La valorisation du capital linéaire est timidement apparue en Grande-Bretagne dans les années 1980, prélude à une belle réussite. Des délaissés ferroviaires y sont alors mués en "voies vertes", des espaces de circulation réservés aux usagers non motorisés, indépendants de la circulation routière, à des fins de loisirs ou dédiés à un usage utilitaire (trajets domicile - école/travail). Les anciennes voies ferrées se révèlent particulièrement adaptées à cet usage : leur largeur, leurs fondations solides et bien drainantes, leurs faibles pentes accueillent aisément cette circulation douce. Les itinéraires relient entre eux des points attractifs du territoire, notamment les villes qu'ils pénètrent à l'abri de la circulation routière, et où ils se raccordent aux transports en commun notamment à hauteur des (anciennes) gares. En Grande-Bretagne plus qu'ailleurs, la multiplicité des voies ferrées désaffectées, que complètent voies de halage ou sentiers le long des cours d'eau, dessinent un véritable maillage (figure 1), à même de créer un vaste réseau de transport alternatif à l'automobile. C'est à une petite association bénévole que l'Angleterre doit la mise en œuvre de ce projet.

\section{A - De Cyclebag à Sustrans}

A l'origine des premières mutations d'infrastructures de transport délaissées en Grande-Bretagne et en Europe, l'association Sustrans est généralement, sinon l'auteur, au moins l'associée de ces valorisations du patrimoine ferroviaire linéaire.

Sustrans (pour Sustainable Transport) est apparue sous le nom de Cyclebag en juillet 1977. En réaction au choc pétrolier, un groupe de cyclistes de Bristol s'engage dans la création d'un réseau de routes dédiées au cyclisme. Loin des bandes cyclables partageant la chaussée avec les automobiles, c'est un réseau de pistes interconnectées et totalement indépendantes du mode routier qu'ils imaginent - à l'image des futures voies vertes. Cyclebag s'appuie dès l'origine sur le réseau de transport abandonné, notamment ferroviaire, bien adapté à leur projet et riche de symboles : il offre, à une infrastructure de transport jugée trop inutile pour perdurer, une nouvelle fonction de déplacement. Sa première réalisation, aujourd'hui encore symbole de Sustrans, est le sentier réalisé sur les $21 \mathrm{~km}$ de l'ancienne voie ferrée menant de Bristol à Bath, fermée sous l'ère Beeching. L'association n'obtient alors l'autorisation que de transformer la section Bitton - Bath en sentier non permanent, sur une largeur de $2 \mathrm{~m}$. Entièrement réalisée par des bénévoles, sa mise en œuvre est longue et l'aménagement initial sommaire. Inauguré dans son intégralité en 1985, le sentier mesure désormais $3 \mathrm{~m}$ de large, est intégralement couvert de bitume et comprend marquage au sol, signalétique, signalisation, mobilier urbain et, à l'instar de nombreuses réalisations de Sustrans, est ponctué d'œuvres d'art contemporain (figure 4).

Forte de ce premier succès, l'association se lance dans d'autres projets. Des négociations abouties avec British Waterways conduisent à un premier élargissement des supports utilisés vers les voies de halage. Cet autre patrimoine du monde des transports offre $3200 \mathrm{~km}$ de sentiers potentiels supplémentaires, dont le premier exemple abouti est le Kennet and Avon Canal cycle route, achevé en 1988. Cet élargissement à d'autres 
emprises renforce le projet de réaliser un réseau cyclable connexe à l'échelle de la Grande-Bretagne, avec en préambule la création d'au moins une route cyclable de qualité dans chaque ville britannique.

\section{B - Un système intégré et visionnaire}

Dès lors, l'association Sustrans a rapidement enchaîné les réalisations. Son succès se mesure aujourd'hui au kilométrage de sentiers réalisés - $19200^{10}$-, au nombre d'adhérents et de sympathisants - 40000 -, à l'étendue nationale des projets en cours, et à son budget annuel de 26 millions de livres. La valorisation et la patrimonialisation des emprises de transport connaissent en Europe des succès divers, au sein desquels émerge la réussite de Sustrans. Trois raisons peuvent être avancées pour l'expliquer.

La première réside dans l'adhésion populaire qui sous-tend chaque projet soutenu par Sustrans. L'association privilégie les projets émanant de collectivités ou d'associations locales auxquelles elle accorde son appui financier et logistique. Communication et concertation, multiplication des réunions publiques, contact personnalisé avec chaque riverain du sentier sont mis en place en amont du projet. Cette volonté d'imposer une bonne gouvernance vise à désamorcer les conflits qui émergent lors de la mutation en lieux publics d'espaces désertifiés. L'impact de cette politique et le respect des éventuels usagers préexistants s'avèrent flagrants, déminant les situations conflictuelles des projets menés sans concertation tels que le GR3 en Indre-et-Loire ${ }^{11}$. Lors du réemploi par Sustrans d'un itinéraire existant (y compris un simple chemin relevant du Right-of-way), contact est pris avec l'ensemble des représentants des utilisateurs, et des solutions adaptées aux différents usages sont envisagées afin de désamorcer les conflits et de s'assurer de l'adhésion populaire au chantier.

Aucune philanthropie n'oriente cette politique, mais une volonté toute pragmatique d'éviter des oppositions longues et coûteuses, et l'assurance de tirer un parti financier du soutien populaire. Son exploitation se traduit notamment par le recrutement de bénévoles qui entretiennent les sentiers, en signalent les dégradations, sensibilisent le public, et organisent des actions de collecte de fonds. L'appui de ces volontaires, localement implantés, aide à l'acceptation du sentier par la population, et en minimise les dégradations. Pour l'association, c'est également un gain financier important : 2500 bénévoles participent non seulement à la gestion et à la promotion du réseau, mais également à sa conception même, la quasi-totalité des travaux étant opérée par eux, ce qui réduit d'autant le coût de conception des sentiers. Enfin, l'adhésion du public au programme de Sustrans lui assure $10 \%$ de ses revenus : ses 40000 supporters lui ont fait don de 2,69 millions de livres en 2007.

La seconde raison de son succès tient dans la cohérence et la connexité du réseau conçu par Sustrans. Dès l'origine, l'association a programmé la réalisation d'un véritable réseau aux mailles connexes, qui irrigue l'ensemble du Royaume-Uni. Elle a ainsi pris une grande longueur d'avance sur le continent, qui multiplie fréquemment les liaisons isolées, avant de tenter de les réunir au sein d'un réseau national. Ainsi, des associations locales, des régions ou des départements français ont valorisé d'anciennes emprises (telle Givry - Cluny en Bourgogne), développant autour d'elles un réseau local, parfois relié à un projet européen ... mais sans référence à un programme national, alors inexistant. Lors de son adoption en 1998, le "schéma national des véloroutes et voies vertes » n'a pu englober l'intégralité de ces réalisations : certaines - 
la voie verte des Hautes-Vosges notamment - s'avèrent géographiquement trop éloignées du réseau imaginé depuis Paris, d'autres - l'Axe Vert de Thiérache entre autres - ne répondent pas aux normes imposées après leur conception. Ces réseaux locaux, nationaux et européens entrent aujourd'hui en concurrence, annihilant toute lisibilité du projet global. La Grande-Bretagne a déjoué cette cacophonie contreproductive, qui dilue les subsides entre des projets sans réelle visibilité, et brouille le message aux yeux du public. Le projet phare de Sustrans, le National Cycle Network (NCN), bénéficie d'une bonne visibilité par son unicité et son envergure nationale (figure 3). De même, la mise en place d'une signalétique et d'une signalisation dédiées et unifiées sur tout le territoire en renforce la cohésion et la reconnaissance. La signalisation s'appuie sur un modèle de numérotation comparable à celui du réseau routier, clairement identifiable en tant que réseau cyclable. Outre l'identification du réseau, le choix de cette signalisation familière favorise sa bonne compréhension, et donc son acceptation de la part des usagers.

Figure 3 - Patrimoine ferroviaire, « railway paths » et « National Cycle Network »

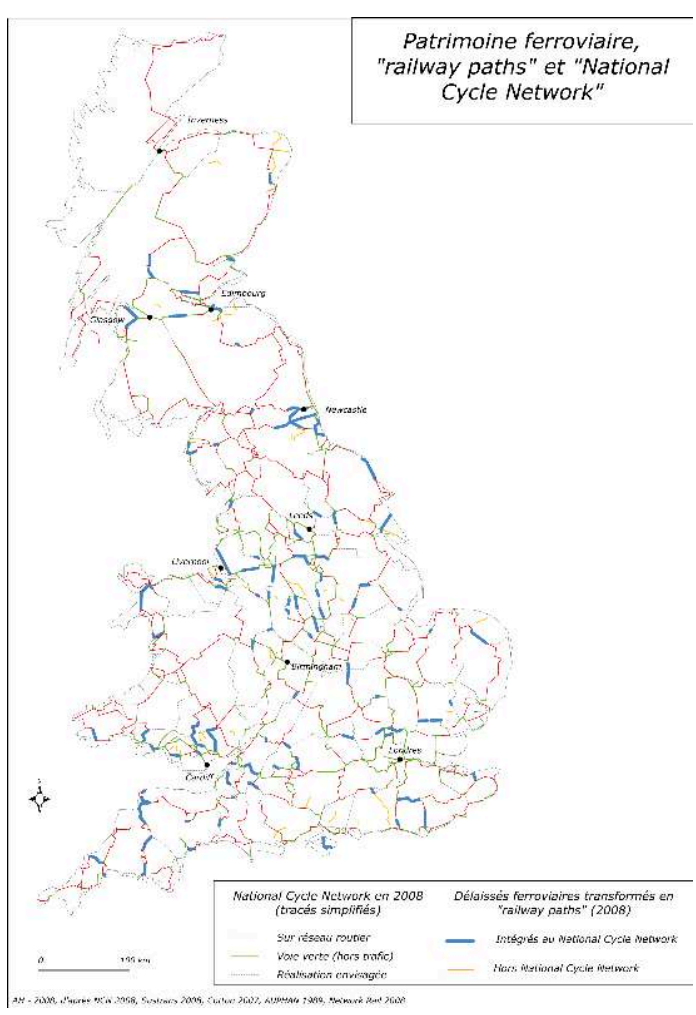

La reconnaissance et l'envergure nationales du NCN ont constitué un troisième élément clef de la réussite de Sustrans. Ainsi, l'adoption en 1996 de la National Cycling Strategy par le Département des Transports, intégrant le NCN au cœur de ses projets, constitue un soutien de poids. Reprenant les mêmes objectifs que le $N C N$, cette politique participe concrètement à sa mise en place, en lui apportant reconnaissance officielle et soutien financier. Cette valorisation nationale a rapidement conduit l'association à se soustraire au cadre restreint des voies vertes, et lui a permis de concrétiser son intégration dans des programmes de plus vaste portée. Les projets de Sustrans ont en effet récemment évolué. Si la constitution du NCN demeure une priorité, d'autres programmes illustrent sa volonté de s'impliquer dans d'autres domaines, et notamment dans la promotion de la santé et la protection de l'environnement. Cette ouverture vers 
d'autres thèmes se traduit notamment par la promotion active du vélo dans la vie quotidienne (programmes Bike it, Active Travel). Pour cela, elle participe activement à la constitution de pistes cyclables, incluse ou non dans son programme phare NCN (safe routes to school, Connect2). Elle s'implique également dans la mutation des espaces urbains sur le modèle des woonerf néerlandaises (Home zones, DIY Streets), qui rééquilibrent l'espace entre modes motorisé et non-motorisé. Dans ce cadre, la prochaine tenue des Jeux Olympiques à Londres a conduit Sustrans à s'impliquer dans la mise en place des sentiers dédiés à la circulation douce des futurs parcs olympiques (GOAL ${ }^{12}$ ), que devrait compléter la réalisation, en collaboration avec Transport for London, d'une nouvelle passerelle sur la Tamise entre Canary Wharf à Rotherhithe, dédiée à la circulation non-motorisée.

Sustrans s'éloigne donc peu à peu de la simple valorisation d'emprises ferroviaires, même si celles-ci trouvent tout naturellement leur place dans ces projets. La question qui pose débat réside toutefois dans l'amenuisement de la part de ce capital au sein de ses programmes. L'évolution de l'association vers de nouveaux idéaux, proches du développement durable, et des choix d'aménagement très pragmatiques conduisent en effet souvent à écarter la valorisation patrimoniale des projets les plus récents.

\section{III - L'oubli du patrimoine}

L'élargissement des activités de Sustrans l'a progressivement amenée à s'éloigner de ses supports originels afin non seulement d'accroître, de manière très pragmatique, son champ d'action, mais également d'adapter ses réalisations à ses nouveaux objectifs : la santé et la sécurité des cyclistes.

\section{A - Le patrimoine ferroviaire aujourd'hui dans les projets de Sustrans}

La première raison d'être de Sustrans résidait dans la mutation d'anciennes voies ferrées en pistes cyclables. Dès 1993, l'association se prévalait ainsi de l'ouverture de $450 \mathrm{~km}$ de sentiers indépendants du trafic routier, prélude à la mise en place d'un véritable réseau autonome devant raccorder entre elles les villes britanniques.

Le changement d'échelle induit par la mise en chantier du NCN en 1995 provoque une évolution radicale des objectifs, des moyens, et de l'esprit même des projets initiés. C'est en effet à un vaste chantier de $10400 \mathrm{~km}$, devant être achevé pour 2005, que l'association s'attaque. Sa réalisation vise à promouvoir le cyclisme comme mode de transport quotidien, à réduire le trafic routier, et à améliorer la santé et l'environnement des britanniques, en réponse aux nouveaux objectifs de l'association. Soutenu et avalisé par le Département des Transports, et bénéficiant de l'adhésion populaire attendue, sa réalisation concrète avance rapidement et de nouvelles tranches sont régulièrement adjointes au projet initial. En 2007, $19200 \mathrm{~km}$ de réseau cyclable ont ainsi été réalisés (figure 3).

Cette exécution rapide a cependant inéluctablement éloigné Sustrans de la valorisation du patrimoine ferroviaire. Les prévisions initiales évoquaient la réalisation du NCN sur des supports variés, incluant $50 \%$ de sites propres, existants ou à créer, notamment d'anciennes voies ferrées valorisées. Le recours annoncé à $50 \%$ d'itinéraires partagés avec le trafic motorisé - rues résidentielles, routes à trafic restreint, ou bandes cyclables sur route plus fréquentée - constituait toutefois une inflexion dans les idéaux 
de l'association. Cette première entorse annihile partiellement l'intérêt du projet en le privant de la sécurité du site propre, et restreint de fait la part des valorisations ferroviaires. Ce taux de mixité va pourtant rapidement s'accroître, puisque aujourd'hui, un-tiers seulement du NCN emprunte des itinéraires indépendants (figure 3).

Ce revirement et le recul du patrimoine ferroviaire se justifient par l'ampleur du projet, revu à la hausse notamment lors de son éligibilité aux projets « Millenium » en 1999, et par la nécessité induite de réaliser rapidement ce réseau très médiatisé. Il est ainsi édifiant d'observer l'évolution du projet : les $50 \%$ de voies vertes initialement prévues représentaient $5200 \mathrm{~km}$, et impliquaient le décuplement des sites propres existants. L'adjonction au NCN de $4000 \mathrm{~km}$ supplémentaires en 1999 ne profite pas à la patrimonialisation, puisque le taux de voies indépendantes tombe alors à $33 \%$, soit un kilométrage inférieur à celui du projet initial $(4750 \mathrm{~km})$. Le réseau routier devient alors le support principal du NCN. Il n'exige en effet qu'un aménagement léger, contrairement à la mutation des emprises ferroviaires, 100 fois plus onéreuses et d'une mise en œuvre plus longue. Seule la route permettait la réalisation rapide et financièrement supportable d'un aussi vaste réseau.

Aujourd'hui, les $19200 \mathrm{~km}$ réalisés ne comprennent que $6400 \mathrm{~km}$ de voies vertes - sans qu'il soit désormais possible d'accéder à des statistiques plus précises concernant la nature des infrastructures employées. La disparition de cette information des bases de données de l'association, associée à la forte baisse de la création de voies vertes, illustrent les évolutions fondamentales de Sustrans, qui délaisse la valorisation patrimoniale au profit d'une vision pragmatique et utilitaire des pistes cyclables.

\section{B - Une vision plus pragmatique que patrimoniale}

Sustrans affiche désormais comme objectif premier la sécurité des cyclistes. Selon elle, la sécurisation de leurs trajets nécessite la multiplication des voies réservées, afin d'amener à terme davantage d'automobilistes à délaisser leur voiture, et à améliorer de fait leur santé. La préoccupation environnementale et sociétale a pris le pas sur la vision patrimoniale du réseau. Pourtant, la mise en œuvre du NCN n'a été rendue possible que par son éligibilité aux projets Millenium, dont les objectifs imposés résidaient dans la valorisation du patrimoine britannique et dans sa préparation à l'entrée dans le XXIès, créant un « héritage durable pour le Royaume-Uni »" ${ }^{13}$. Pourtant, peu à peu, la valorisation patrimoniale s'est effacée devant une mise en œuvre plus pragmatique du réseau.

En effet, seule une bonne fréquentation justifie la multiplication des voies vertes ou des pistes cyclables. Or pour qu'elles soient attractives, il apparaît nécessaire que les trajets soient les plus rapides et directs possibles. Ce tracé idéal est alors imaginé par Sustrans (ou les collectivités locales qu'elle soutient) sur le papier, avant que ne soit concrètement cherché sur le terrain les moyens de le réaliser efficacement et économiquement. Dès lors, toute emprise de transport désaffectée, idéalement placée sur son tracé, est intégrée au projet, et ce en dépit du surcoût notoire qu'elle induit. La stricte séparation vis-à-vis de la circulation motorisée s'avère en effet décisive dans le succès des sentiers: le rapport annuel $2007 \mathrm{du} N C N$ estime ainsi que les sections ségrégées du trafic routier concentrent à elles seules $82 \%$ des trajets enregistrés sur le $N C N$, alors qu'elles ne représentent qu'un-tiers de ce dernier. Néanmoins, ce surcroît de fréquentation s'avère nul si l'itinéraire impose un détour par rapport à la route. Cela 
conforte Sustrans dans sa volonté de réaliser les trajets les plus directs possibles, abandonnant les emprises ferroviaires au profit d'une implantation sur chaussée partagée avec l'automobile.

Par ailleurs, la nécessité de réaliser ce réseau rapidement et à faible coût écarte fréquemment les anciennes voies ferrées des projets. Ainsi, la réalisation d'une section de la route $N C N n^{\circ} 66$, entre Beverley et Market Weighton au nord-ouest de Hull, a conduit à un choix étonnant: une ancienne voie ferrée en bon état, reliant ces deux communes et déjà fréquemment utilisée par les amateurs de vélo tout terrain (Hudson Way), a été écartée au profit d'une création sur route à circulation partagée, au prix pourtant de quelques détours. La volonté de desservir quelques villages, à l'écart de l'ancienne emprise ferroviaire, a pu être évoquée. Mais c'est de manière très claire qu'a été énoncé par Sustrans le principal désavantage de l'ancienne voie ferrée : dénuée de sa couverture de ballast, elle était devenue boueuse, et nécessitait un aménagement spécifique. La route, quant à elle, était immédiatement disponible, et ne nécessitait comme investissement que la sécurisation des grands axes, et la mise en place de la signalétique et de la signalisation.

Ces choix s'opposent doublement aux opérations recensées sur le continent européen notamment en Espagne, en Allemagne ou en Belgique - et aux Etats-Unis. La mise en œuvre des réseaux verts s'y appuie prioritairement sur les anciennes emprises de transport, qu'ils ont pour objectif de valoriser. Le bilan des délaissés disponibles et de leur état donne lieu au dessin du futur réseau vert, établi en fonction de leur emplacement et des connexions possibles entre elles. Des sections de sentiers à créer ex nihilo, voire exceptionnellement des sections de routes très calmes, sont tolérées de manière minoritaire pour assurer la jonction entre deux délaissés. Davantage que son utilité, c'est donc la valorisation du patrimoine qui est recherchée à travers le réseau vert. La préservation de la mémoire de ses anciennes fonctions à travers le nom, la signalétique, ou le "décor» de la voie verte illustrent ce souci patrimonial. S'il se retrouve dans le nom des premiers aménagements de Sustrans, comme le Bristol \& Bath Railway Path, il n'apparait plus clairement dans les nouveaux sentiers. Considérés à petite échelle, ils mêlent au long de leur parcours de multiples supports, et leurs dénominations, assez neutres, ne font plus appel à leurs origines.

Par ailleurs, les réalisations américaines et continentales s'accordent sur la valorisation des emprises dans leur intégralité, tout du moins dans leurs parties réutilisables. A l'inverse, le pragmatisme britannique s'attache à ne réemployer du délaissé que la section qui lui est utile, sans s'encombrer du reste de l'infrastructure. Ainsi que l'illustre la figure 3, de nombreuses sections courtes, souvent inférieures à $10 \mathrm{~km}$, sont ainsi prélevées et intégrées à une réalisation de Sustrans, le reste de l'emprise étant abandonné. Entre autres exemples, on peut citer Willow Walk (route $\mathrm{NCN}^{\circ}{ }^{\circ}$ ), qui emprunte ponctuellement 8 des $183 \mathrm{~km}$ que compte l'ancienne voie ferrée du Somerset and Dorset Joint Railway. Ce court tronçon, à l'ouest de Glastonbury, dessine un raccourci à travers un espace naturel sensible, le Shapwick Heath Nature Reserve, que la route traditionnelle contourne. Une fois cet espace protégé traversé, le NCN s'écarte du tracé rural de l'ancienne voie ferrée, et rejoint à Shapwick la route principale vers Bridgewater, bordée de plusieurs villages.

Ces courtes sections sont certes sauvegardées et mises au service de la circulation douce. Mais pour l'infrastructure dans son entier, elles représentent davantage un mitage qu'une réelle valorisation, tant le prélèvement d'une section, notamment 
centrale, constitue un handicap majeur à tout autre réemploi. Il participe de la destruction des principales valeurs patrimoniales de l'infrastructure, sa linéarité et sa continuité, qui favorisaient la mise en relation de différents points du territoire. Cet éloignement de la démarche patrimoniale est d'ailleurs totalement assumé par Sustrans, qui revendique la primauté de ses nouveaux objectifs, la santé et l'environnement, sur une quelconque préservation d'un héritage, devenu à ses yeux très secondaire.

\section{C - Un réseau utilitaire avant tout} réservées et indépendantes, notamment en milieu urbain où la densité de circulation rend la mixité dangereuse. Les anciennes emprises de transport offrent la possibilité d'une telle ségrégation des usagers. Leur présence, dans un environnement urbain où l'espace disponible s'avère rare, onéreux et de continuité aléatoire, constitue en outre un atout essentiel. Sustrans, lorsqu'elle ne peut créer d'espace dédié aux seuls cyclistes, emploie les parcs urbains et les rues résidentielles, où elle œuvre à la mise en place de mesures destinées à "calmer le trafic »: la cohabitation cyclistes/voitures est jugée acceptable en-dessous de 900 véhicules/h roulant à la vitesse maximale de $30 \mathrm{~km} / \mathrm{h}$. Cette réappropriation des rues, au sein de quartiers qui replacent le piéton et le cycliste au centre de l'espace (Home Zones) lui semble plus bénéfique que la réalisation de pistes cyclables sur rue partagée. caractère utilitaire particulièrement marqué, eu égard au pragmatisme de l'association. Pour développer efficacement l'usage du vélo, elle axe en effet son action sur les fortes concentrations de population, où les cyclistes potentiels sont les plus nombreux, et où chaque intervention rencontre le meilleur écho. Par ailleurs, pour améliorer l'image du vélo via une pratique sécurisée, priorité est donnée aux aménagements en centre-ville, où le cyclisme est particulièrement dévalorisé du fait de sa dangerosité. Dès lors, les rares emprises abandonnées en milieu urbain, et notamment en entrée/sortie de villes, sont mises à contribution. Les sorties d'Edimbourg, ou la traversée de Newcastle-UponTyne sont ainsi desservies par des voies vertes, implantées sur des emprises ferroviaires ou des bords de rivière. Néanmoins, en secteur urbain aussi, l'entreprise de valorisation demeure très ponctuelle - à l'instar des $4,8 \mathrm{~km}$ prélevés à Innocent Railway à l'est d'Edimbourg, ou des courtes sections ferroviaires ponctuant les $14 \mathrm{~km}$ de Hadrian Cycleway à travers Newcastle. Là encore, seule la mise à disposition des circuits sécurisés a été prise en compte, au prix de la dévalorisation des vastes emprises ainsi démembrées.

Les mutations urbaines mettent en lumière un antagonisme essentiel entre les exemples continentaux et les choix de Sustrans. L'association britannique ne mise que sur la réalisation de sentiers utilitaires : ils permettent de se rendre en ville, au travail, à l'école, à un espace de loisirs. En revanche, leur potentiel purement récréatif (le vélo en tant que loisir) est totalement éclipsé par cette recherche d'utilité quotidienne. Ainsi, le bilan annuel ${ }^{14}$ de Sustrans fait état de 338 millions de trajets recensés sur le réseau $N C N$, dont $1 / 3$ de très courtes distances, de l'ordre du lien local et quotidien. Sur 
le continent, ces trajets quotidiens sont rares: en France, les $2 / 3$ des pratiquants parcourent de 15 à $35 \mathrm{~km}$ par trajet, et seuls $13 \%$ fréquentent les voies vertes plus d'une fois par semaine ${ }^{15} \ldots$ Si elles endossent également un rôle utilitaire, leur vocation est davantage orientée vers un usage récréatif $(92 \%$ contre $8 \%$ pour la pratique utilitaire). Elles s'appuient sur la même volonté de sécuriser la pratique cycliste mais elles s'inscrivent dans un cadre plus volontiers rural qu'en Grande-Bretagne.

leurs voies vertes, les Etats europeens visent certes à valoriser un patrimoine ferroviaire, mais également à revitaliser leurs espaces ruraux. Excursionnistes et cyclotouristes consomment et induisent de nouveaux emplois, aux apports économiques non négligeables pour les régions concernées. Les retombées économiques du Donauradweg sont notamment estimées à 7,5 millions d'euros pour la seule Haute-Autriche. Les Vias verdes espagnoles participent à rééquilibrer la pression touristique vers les espaces intérieurs du pays, qui enregistrent sur leur passage un regain de vitalité1 ${ }^{16}$. A l'inverse, le réseau britannique minimise ce potentiel touristique. Le caractère urbain des espaces desservis l'atteste. L'aménité des paysages et les dessertes rurales, plébiscitées par les randonneurs, sont négligées. La cohabitation bande cyclable/circulation routière, quasi systématique en milieu rural, s'oppose également à la politique des véloroutes et des voies vertes, qui valorisent les sites propres, plus attractifs. Enfin, l'absence délibérée d'équipements et de services spécifiques à cette clientèle - hôtellerie, restauration rapide, réparation de vélos, portage de bagage, fléchage touristique, boucles cyclables ...- qui s'étirent habituellement au long des voies vertes, illustrent le parti-pris britannique d'ignorer le potentiel touristique des pistes cyclables.

Figure 4 - L'entrée de la voie verte Bristol \& Bath Railway Path 
Première réalisation de Sustrans, alors encore dénommé Cyclebag, le Bristol \& Bath Railway Path constitue, aujourd'hui encore, le symbole de l'association. Depuis son inauguration (partielle) en 1984 , son aménagement s'est grandement amélioré : élargissement du sentier jusqu'à $3 \mathrm{~m}$, pose de bitume, d'éclairage public, de signalisation, d'une signalétique et d'une numérotation des itinéraires unifiées à l'échelle du territoire britannique, de mobilier urbain, d'élements de sécurisation..

L'implantation d'oeuvres d'art, généralement des sculptures, au long des parcours, participe de l'image des sentiers de Sustrans. A l'occcasion du 30è anniversaire de l'association en 2007, l'ancienne porte en béton marquant l'entrée de ce sentier symbolique a été remplacée par cette sculpture métallique, "Twisted Arch", qui symbolise la croissance de l'association, et la régénération des espaces abandonnés.

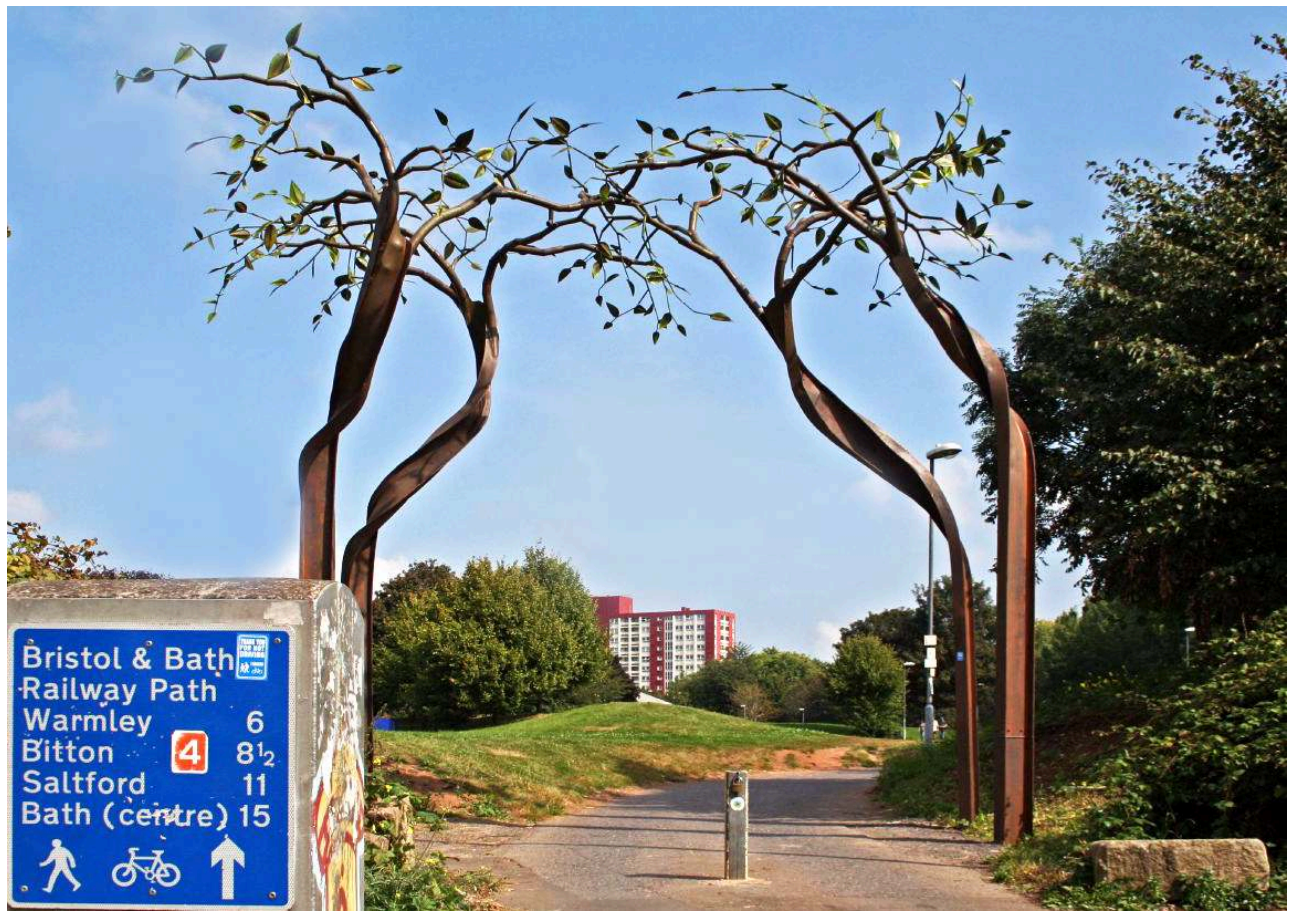

La pratique récréative et touristique endosse alors non pas le rôle d'objectif final qui est le sien sur le continent, mais celui de moyen d'action. Aussi la participation de Sustrans au projet pan-européen d'Avenue Verte Londres - Paris est-elle moins considérée par l'association comme une source de revenus et de développement potentiels, que comme l'une de ses stratégies marketing pour asseoir la réussite de son réseau: la pratique du vélo durant les vacances sensibiliserait les vacanciers britanniques à ce mode de déplacement, et favoriserait sa banalisation dans la vie quotidienne. Même la traditionnelle mise en place d'œuvres d'art au long des sentiers (figure 4) appartient désormais à ces stratégies : l'art permet «d'utiliser d'autres arguments et d'autres médias afin de porter [le] message [de Sustrans] auprès de ceux qui ne s'intéresseraient jamais aux questions de transport, d'environnement ou d'urbanisme $»^{17}$. Il permet notamment d'inciter des personnes, peu sensibilisées à la pratique du vélo, à fréquenter les pistes cyclables, dans l'espoir de les fidéliser, mais aussi d'attirer largement l'attention sur cette cause à ses yeux primordiale. Pour Sustrans, les "voies vertes » focalisent tout l'espoir d'une société plus vivable et d'un environnement plus « durable », annoncés dans le nom même de l'association.

\section{Conclusion}

En Grande-Bretagne, la préservation et la valorisation de l'héritage issu du démantèlement drastique du réseau ferré national s'opère à la discrétion de structures 
privées, sans réel contrôle de l'Etat, à l'image du libéralisme quasi-absolu qui avait prévalu lors de sa constitution. Certes, un arsenal de mesures est venu protéger le patrimoine bâti et monumental, et notamment certains ouvrages d'art. Mais, davantage encore que sur le continent européen, le capital des infrastructures linéaires reste dans l'ombre, confié, presque par hasard, aux seules mains d'une association sensibilisée à son potentiel. Aujourd'hui, face à l'évolution des objectifs de cette dernière, ce choix peut s'avérer discutable, d'autant que si quelques autres initiatives privées ont pu prendre le relais localement, il leur manque la reconnaissance et les moyens de Sustrans pour établir de véritables réseaux cohérents.

50 Loin d'une quête patrimoniale, Sustrans s'est donc recentrée sur des programmes nationaux environnementaux et sociétaux, sans rapport avec le patrimoine ferroviaire. Néanmoins, un projet très récent montre un léger infléchissement de la politique de l'association. Les grands itinéraires du NCN, largement routiers et dessinés à l'échelle nationale, cohabitent désormais avec les courts projets de CONNECT2, élus en 2007. Ils ciblent très localement les nécessités de ( $r$ )établir un passage piétonnier ou cycliste impossible, ainsi que les possibilités très concrètes d'y répondre. Outre l'échelle beaucoup plus locale de la réflexion et la taille restreinte des projets, on assiste là au retour des voies vertes: emplois de différents supports, notamment d'anciennes voies ferrées, et indépendance vis-à-vis du mode routier.

51 Toutefois, si CONNECT2 semble marquer un retour vers la valorisation patrimoniale, ce programme n'en demeure pas moins une menace pour le capital ferroviaire. En effet, le pragmatisme de l'association reste de mise, et conduit toujours au mitage des emprises employées, à l'image du projet du Afon Tawe Bridge, entre Clydach et Glais (NE de Swansea): la réouverture de cet ancien pont ferroviaire rétablit la jonction des quartiers de part et d'autre de la rivière Tawe et de leurs voies vertes respectives, déjà existantes. Le réemploi ferroviaire se limitera donc à moins d'un demi-mile. Cet exemple représentatif des nouveaux projets de Sustrans ne permet pas de réellement espérer que le patrimoine des anciennes infrastructures de transport, notamment ferroviaires, tire davantage de bénéfice de ce programme que des précédents.

Dès lors, le risque est grand de voir à court terme disparaître une part essentielle de cet héritage britannique, mité et partiellement fondu au sein de valorisations plus vastes, sans mémoire de son passé. Le risque excède toutefois la Grande-Bretagne, l'une des deux portes d'entrée du processus de valorisation des voies vertes sur le continent européen. Si les modèles belge et espagnol, qui s'appuient sur la préservation patrimoniale, demeurent prioritaires, le modèle britannique fait des émules, notamment en France et à l'échelle pan-européenne. A terme, c'est donc une part essentielle du capital linéaire des délaissés ferroviaires qui pourrait disparaitre, victime du projet qui devait, primitivement, assurer sa pérennité. 


\section{BIBLIOGRAPHIE}

AUPHAN E. (1989). - Obsolescence ou renaissance des réseaux ferrés pour le transport de voyageurs en Europe occidentale? (Grande-Bretagne, France, RFA), Aix-en-Provence, Thèse présentée pour le doctorat ès-Lettres et sciences humaines, $1491 \mathrm{p}$.

Association pour l'Histoire des chemins de fer en France (2001). - « Le Patrimoine ferroviaire : enjeux, bilans et perspectives ", Actes du $6^{\mathrm{e}}$ colloque de l'AHICF (Mulhouse 23-26 septembre 1998), Revue d'Histoire des Chemins de fer, $\mathrm{n}^{\circ}$ 20-21.

BRB (Residuary) Limited (2007). - Annual Report on Built Heritage 2006-2007, 13 p., accessible en ligne : http://www.brb.gov.uk/railwayheritage/heritage-annual-report-2006-2007

BURMAN P., STRATTON M. (1997) - Conserving the Railway heritage, Londres, Taylor \& Francis Group, $244 \mathrm{p}$.

COTTON N. (2007). - Traffic-free cycle trails, cycle-city guides, $384 \mathrm{p}$.

Cotton N., Grimshaw J. (2005). - Cycling in the UK, the official guide to the National Cycle Network, Sustrans, $303 \mathrm{p}$.

HECKER A. (2004). - Quelles valorisations pour les voies ferrées désaffectées ? Le cas de la Lorraine, thèse présentée pour le doctorat de géographie, Paris IV-Sorbonne, $584 \mathrm{p}$.

HECKER A. (2006). - « Véloroutes et voies vertes : supports, ou objets touristiques ? », Cahiers de Géographie, n 4-2006, p. 199-208

Insall Ph. (2003) - «Sustrans au Royaume-Uni : des projets démonstratifs pour les transports durables ». In : Vélo : de l'image à l'usage ..., Actes des Rencontres du Club des Villes Cyclables, Paris, 2 avril 2003, $27 \mathrm{p}$.

MERCAT N., Bureau ALTERMODAL (2003). - Voies vertes : fréquentation et impact, AFIT, édition Panorama de l'offre, $93 \mathrm{p}$.

Millenium Commission (2007) - A lasting legacy from your lottery funding, brochure

National Railway Museum, Annual Review 2006-2007, 36 p., accessible en ligne : http:// www.nrm.org.uk/visitor/annualreview.asp

ODIT F. (2006). - France à vélo, France des voies vertes, les clefs de la réussite, ODIT, collection

Ingénierie touristique, $104 \mathrm{p}$.

SERMENTO J. (2002). - "The Geography of "disused" railways : what is happening in Portugal?", Finisterra, XXXVII, p. 55-71

SUSTRANS (2007). - Annual Review, disponible en ligne : http://www.sustrans.org

SUSTRANS (2007). - Annual Monitoring Report on the National Cycle Network, disponible en ligne :

http://www.sustrans.org

VESCHAMBRE V. (2008). - Traces et mémoires urbaines, enjeux sociaux de la patrimonialisation et de la destruction, Rennes, Presses Universitaires de Rennes, 315 p. 


\section{NOTES}

1. Réseaux voyageurs et marchandises, hors LGV.

2. $31000 \mathrm{~km}$ de voies ferrées sont officiellement en service au Royaume-Uni (Network Rail). Cette donnée correspond au kilométrage des lignes de chemins de fer multiplié, pour chaque itinéraire, par le nombre de voies qui le composent. Les $17067 \mathrm{~km}$ de liaisons ferrées déduisent le réseau hors Grande-Bretagne, et ne prennent en compte chaque itinéraire qu'une seule fois, indépendamment du nombre de voies ferrées qui le composent.

3. Seule celle ayant subsisté est représentée sur la carte 1 . Sur l'ensemble du document, la trop grande proximité de certaines emprises n'a pas permis la représentation de la totalité des voies redondantes.

4. Comment concurrencer efficacement la route, quand on dispose, en 1962, d'un parc de traction encore constitué à $70 \%$ de locomotives à vapeur, alors qu'il n'en reste déjà plus que $41 \%$ en France?

5. National Railway Museum Review 2006 - 2007

6. "Ancient Monument and Archeological Areas Act", 1979

7. Que l'on pourrait comparer à nos bâtiments «inscrits ». Ils relèvent du «Planning (Listed Buildings and Conservation Areas) Act " de 1990 pour l'Angleterre et le Pays de Galles, et du « Town and Country Planning (Listed Buildings and Conservation Areas) (Scotland) Act » de 1997 pour l'Ecosse.

8. "Areas of special architectural or historic interest, the character or appearance of which it is desirable to preserve or enhance", "Planning (Listed Buildings and Conservation Areas) Act" de 1990.

9. BRB (Residuary) Limited, 2007

10. Ces données, issues du rapport annuel de Sustrans (Sustrans, 2007) correspondent au bilan à la fin de l'année 2007.

11. La mutation par le Conseil Général d'un sentier rural de la FDRP en véloroute bituminée s'est opérée sans que la FDPR n'en soit avertie, induisant une levée de bouclier contre cet aménagement.

12. GOAL, pour Greenways for Olympics And London

13. Millenium Commission, 2003

14. Sutrans, 2007

15. MERCAT et Intermodal, 2003

16. Pour ces deux exemples, voir HECKER, 2006

17. INSALL, 2003

\section{RÉSUMÉS}

Aux $\mathrm{XIX}^{\mathrm{e}}$ et $\mathrm{XX}^{\mathrm{e}}$ siècles, la Grande-Bretagne a assisté à l'intense contraction d'un maillage ferroviaire pléthorique, la laissant à la tête d'un vaste patrimoine hérité. Si les bâtiments et les ouvrages d'art bénéficient d'une reconnaissance patrimoniale, le capital linéaire souffre d'un manque réel de patrimonialisation.

La Grande-Bretagne, à travers l'association Sustrans, a pourtant introduit en Europe le concept de voies vertes, qui valorise les infrastructures sous forme de sites propres réservés aux circulations non-motorisées. Rapidement toutefois, des divergences notables ont singularisé les 
réalisations britanniques. Face à des réalisations continentales à vocation patrimoniales, essentiellement publiques, majoritairement éparses et globalement dédiées au tourisme, la Grande-Bretagne oppose un véritable réseau maillé, continu et privé, à destination utilitaire, et qui relègue le tourisme, mais également désormais la préservation des infrastructures, à la marge de vastes projets de « développement durable ».

La crainte est donc désormais de voir disparaître une part essentielle de cet héritage, victime du projet qui devait, primitivement, assurer sa pérennité.

In the nineteenth and twentieth centuries, Great Britain underwent a severe contraction of a rich railway network, thus creating a vast heritage. While buildings and structures are recognized assets, railway lines lack real recognition as heritage. Britain, thanks to the Sustrans charity, has introduced in Europe the concept of greenways, which promotes infrastructure as a site reserved for specific non-motorized traffic. Soon, however, significant differences have singled out the British achievement. While the continental approach is mostly public, scattered and generally dedicated to tourism, Britain has maintained a truly meshed network, with a continuous, private, and utilitarian purpose. It does relegate tourism, but also heritage preservation of infrastructure, on the fringe of large "sustainable development" projects. The fear now is of losing an essential part of this legacy, a victim of the project which was, originally, to ensure its sustainability.

Im 19 und $20 \mathrm{Jh}$. hat das weit ausgedehnte Eisenbahnnetz in Großbritannien stark geschwunden, so dass ein weites Erbe entstand. Wenn die Gebäude und die Ingenieurbaue als Denkmäler anerkannt werden, leidet das Erbe aus Linien unter einer Mangel von Pflege.

Mit dem Verein Sustrans hat jedoch Großbritannien das Konzept von grünen Wegen in Europa eingeführt, das die Linien als Standort für nicht motorisierten Verkehr aufwertet. Die britischen Werke kennzeichnen sich durch Divergenze. Im Gegensatz zu dem Kontinent, wo die ehemaligen Bahnlinien vereinzelt öffentlich und für touristische Nutzung sind, entstand in Großbritannien ein dichtes privates Netz aus durchgehenden Linien, in dem Fremdenverkehr sowie der Unterhalt der Infrastrukturen eine Nebenrolle zugunsten großer Projekte nachhaltiger Entwicklung spielt. Es ist zu fürchten, dass ein großes Teil dieses Erbes als Opfer des Projektes verschwindet, das sein Weiterbestehen sichern sollte.

\section{INDEX}

Schlüsselwörter : Eisenbahnerbe, Erbe, grüne Wege, stillgelegte Bahnlinie, Sustrans

Keywords : declassified infrastructures, greenways, heritage, railway heritage, Sustrans

Mots-clés : héritage, infrastructure déclassée, patrimoine ferroviaire, patrimonialisation, Sustrans, voies vertes

\section{AUTEUR}

\section{ANNE HECKER}

Maître de conférences en géographie - Université Nancy 2 - EA 1135 Centre d'Etudes et de Recherches sur les Paysages 